\title{
Short communication: Accounting for new mutations in genomic prediction models
}

\author{
Joaquim Casellas, ${ }^{* 1}$ Cecilia Esquivelzeta, ${ }^{*}$ and Andrés Legarra† \\ *Grup de Recerca en Remugants, Departament de Ciència Animal i dels Aliments, Universitat Autònoma de Barcelona, 08193 Bellaterra, Spain \\ †INRA, UR631 SAGA, F-31326 Castanet Tolosan, France
}

\begin{abstract}
Genomic evaluation models so far do not allow for accounting of newly generated genetic variation due to mutation. The main target of this research was to extend current genomic BLUP models with mutational relationships (model AM), and compare them against standard genomic BLUP models (model A) by analyzing simulated data. Model performance and precision of the predicted breeding values were evaluated under different population structures and heritabilities. The deviance information criterion (DIC) clearly favored the mutational relationship model under large heritabilities or populations with moderate-to-deep pedigrees contributing phenotypic data (i.e., differences equal or larger than 10 DIC units); this model provided slightly higher correlation coefficients between simulated and predicted genomic breeding values. On the other hand, null DIC differences, or even relevant advantages for the standard genomic BLUP model, were reported under small heritabilities and shallow pedigrees, although precision of the genomic breeding values did not differ across models at a significant level. This method allows for slightly more accurate genomic predictions and handling of newly created variation; moreover, this approach does not require additional genotyping or phenotyping efforts, but a more accurate handing of available data.
\end{abstract}

Key words: accuracy, genomic selection, mutation, relationship matrix

\section{Short Communication}

Current availability of massive genotyping technologies based on SNP has provided high-throughput data for the prediction of genomic breeding values by appropriate analytical approaches (Meuwissen et al., 2001). These genomic breeding values try to capture the additive genetic variability from QTL, although recent studies suggested that part of the genetic variability

Received December 11, 2012.

Accepted April 22, 2013.

${ }^{1}$ Corresponding author: joaquim.casellas@uab.cat could escape inclusion in genomic predictions (Maher, 2008). Indeed, Casellas and Varona (2011) showed that the statistical performance of genomic prediction models was highly influenced by both the selective history of the trait and the age of each mutation involved in a given QTL, recent mutations systematically escaping inclusion in analyses. Note that genomic analyses would typically not take into account the effect of new mutations. Genomic BLUP (GBLUP) models (NejatiJavaremi et al., 1997) account for additive genetic relationships by the genomic relationship matrix $(\mathbf{G}$; VanRaden, 2008). Despite the expectation of $\mathbf{G}$ being the regular numerator relationship matrix $(\mathbf{A})$, several authors highlighted the gain by using $\mathbf{G}$ within the context of animal breeding (Legarra et al., 2008; VanRaden et al., 2009). Nevertheless, $\mathbf{G}$ does not properly account for new mutations contributing novel additive genetic variability after the founder generation. This limitation was overcome by Wray (1990) for the standard matrix $\mathbf{A}$, and the main objective of this short communication was to adapt and evaluate the approach of Wray (1990) and Casellas and Medrano (2008) into a genomics context.

Although $\mathbf{G}$ can be constructed by several different methods (VanRaden, 2008), we focused as an example on the first approach outlined by VanRaden (2008), given the benefits reported by Toro et al. (2011). Nevertheless, our approach can be straightforwardly adapted to other algorithms for $\mathbf{G}$. In this research, procedures for calculating $\mathbf{G}$ were adapted to accommodate the occurrence of new mutations in the autosomal genome [i.e., the mutational genomic relationship matrix $\left.\left(\mathbf{G}_{m}\right)\right]$. Note that $\mathbf{G}_{m}$ can be defined as $\mathbf{G}_{m}=\mathbf{G}_{1}+\mathbf{G}_{2}$ $+\ldots+\mathbf{G}_{g}, \mathbf{G}_{q}$ being a genomic relationship matrix where individuals from generation $q$ were treated as founders and the rows and columns linked to ancestors from previous generations were fixed to 0 . Assuming $\sigma_{m}^{2}$ as the mutational variance, $\mathbf{G}_{q} \sigma_{m}^{2}$ must be viewed as the variance-covariance matrix of additive genetic effects attributed to mutations arising in generation $q$. To avoid the successive computation of all intermediate $\mathbf{G}_{q}$ matrices, the algorithm described by Casellas (2011) can be applied. 
Two different hierarchical mixed linear models were assumed and alternative parameterization of mixed model equations by Henderson (1984) was adopted to avoid the inversion of matrices $\mathbf{G}$ and $\mathbf{G}_{m}$. The standard GBLUP parameterization $(\operatorname{model} \mathbf{A})$ was $\mathbf{y}=\boldsymbol{\mu}$ $+\mathbf{Z}\left(\mathbf{G a}^{\bullet}\right)+\mathbf{e}$, where $\mathbf{y}$ was the vector of phenotypic records, $\boldsymbol{\mu}$ was the vector of population means, e was the vector of residuals, and $\mathbf{a}=\left(\mathbf{G a}^{*}\right)$ was the vector of additive genomic effects with appropriate incidence matrix Z . From a Bayesian point of view, the conditional distribution of $\mathbf{y}$ was assumed multivariate normal $\left\{\right.$ i.e., $\left.M V N\left[\boldsymbol{\mu}+\mathbf{Z}\left(\mathbf{G a}^{\bullet}\right), \mathbf{I} \sigma_{e}^{2}\right]\right\}$, and the a priori distribution of $\mathbf{a}^{\bullet}$ was $p\left(\mathbf{a}^{\bullet} \mid \mathbf{G} \sigma_{a}^{2}\right) \sim M V N\left(\mathbf{0}, \mathbf{G}^{-1} \sigma_{a}^{2}\right)$. Note that $\mathbf{I}$ was an incidence matrix with dimensions equal to the number of elements in $\mathbf{y}$, and $\sigma_{a}^{2}$ and $\sigma_{e}^{2}$ were additive genomic and residual variances, respectively. Remaining a priori distributions were assumed flat with appropriate boundaries. Estimated allelic frequencies in the base generation were used to compute $\mathbf{G}$, as used in North American dairy cattle genomic evaluations (P. M. VanRaden, Animal Improvement Programs Laboratory, ARS, USDA, Beltsville, MD, personal communication). On the other hand, an expanded version of the previous model (model AM) was proposed to account for new mutational genomic effects $(\mathbf{m})$ as follows: $\mathbf{y}=$ $\boldsymbol{\mu}+\mathbf{Z}\left(\mathbf{G a}^{\bullet}\right)+\mathbf{Z}\left(\mathbf{G}_{m} \mathbf{m}^{\bullet}\right)+\mathbf{e}$, where the conditional distribution of $\mathbf{y}$ was assumed $M V N\left[\boldsymbol{\mu}+\mathbf{Z}\left(\mathbf{G a}^{\bullet}\right)+\mathbf{Z}\left(\mathbf{G}_{m} \mathbf{m}^{\bullet}\right), \mathbf{I} \sigma_{e}^{2}\right]$, and a priori distributions for genomic effects were $\operatorname{MVN}\left(\mathbf{0}, \mathbf{G}^{-1} \sigma_{a}^{2}\right)$ and $\operatorname{MVN}\left(\mathbf{0}, \mathbf{G}_{m}^{-1} \sigma_{m}^{2}\right)$. As for model A, the remaining a priori distributions were assumed flat.

Simulations relied on a diploid genome composed by 10 chromosomes (100 cM each) with 2,000 SNP and 200 QTL per chromosome. Each replicate evolved during 1,000 non-overlapping generations with effective size $\left(\boldsymbol{N}_{e}\right)$ 100. Founder individuals were homozygous throughout the whole genome for the wild-type allele (i.e., allele 1), and mutation rates of $2.5 \times 10^{-3}$ (SNP) and $2.5 \times 10^{-5}$ (QTL; Meuwissen et al., 2001) were applied during the first 1,000 generations, switching the allele state from 1 to 2 , or vice versa. After that, generations expanded to 500 individuals, with 50 males and 450 females under random mating; mutation rate for SNP changed to $2.5 \times 10^{-8}$ (Hickey and Gorjanc, 2012), whereas this parameter did not modify for QTL markers. Analyses were performed on 3 different scenarios with 3 (G3), 5 (G5), or 10 (G10) generations with both genomic (i.e., genotypes from polymorphic SNP) and phenotypic data (see below), and a last generation only contributing genomic data. Although the number of generations was a rough simplification of the real structure of current livestock populations, scenario G3 could be viewed as a reasonable approximation to a long-generation interval population with few generations contributing data (e.g., dairy cattle), whereas scenarios G5 and G10 tried to mimic medium- (e.g., intensive pig breeds) and short-generation interval populations (e.g., rabbits and broilers), respectively. On the other hand, 3 different heritabilities $\left(h^{2}\right)$ were assumed for the phenotypic trait $\left(\mathrm{h}^{2}=0.1,0.25\right.$ and 0.5 ), with $\sigma_{e}^{2}=1$, and QTL effects were appropriately scaled. Note that the wild-type QTL had a null contribution to the phenotype, whereas the additive effect of the mutate allele was sampled from a gamma distribution with shape and scale parameters 0.4 and 1.6 (Meuwissen et al., 2001). Moreover, the sign of every QTL effect was sampled to be positive or negative with probability 0.5. A total of 100 replicates were analyzed for each scenario and heritability. Model performance was evaluated in terms of goodness of fit by the deviance information criterion (DIC) statistic (Spiegelhalter et al., 2002) and the precision of predicted breeding values ( $\mathbf{u})$, being $\mathbf{u}=\mathbf{a}($ model $\mathrm{A})$ or $\mathbf{u}=\mathbf{a}+\mathbf{m}$ (model AM). Precision was calculated for each replicate as the correlation coefficient $\left(\mathbf{r}_{u, \hat{u}}\right)$ between simulated and predicted breeding values. Within a Bayesian framework, all replicates were analyzed under model $\mathrm{A}$ and model AM. For each analysis, a unique Monte Carlo Markov chain with 100,000 iterations was launched, after discarding the first 10,000 iterations as burn-in (Raftery and Lewis, 1992).

Given that this research focused on the analysis of simulated populations, a preliminary examination of the estimated $\sigma_{m}^{2}$ became mandatory to corroborate the validity of further results about model performance. As shown in Table 1, estimated mutational heritabilities $\left(\mathrm{h}_{m}^{2}=\sigma_{m}^{2} / \sigma_{T}^{2}\right.$, where $\sigma_{T}^{2}$ is the total phenotypic variance) averaged between 0.04 and $0.25 \%$, agreeing with the range of values previously reviewed by Lynch (1988) and Houle et al. (1996); indeed, even the most extreme estimates fit the range, although some of them were close to the lower boundary. This highlighted the conservative behavior of the simulation procedure where the impact of new mutations was not artificially inflated, preventing unrealistic advantages for the model from accounting for new mutational variance.

Both GBLUP models were compared in terms of goodness of fit by the DIC statistic, which suggested important influences from heritability and pedigree depth (Table 2). Note that the DIC evaluates both model fit and complexity, and average differences larger than 3 to 5 DIC units are generally assumed statistically relevant (Spiegelhalter et al., 2002). Small heritabilities and shallow pedigrees favored model A, whereas 
Table 1. Estimated heritabilities under the different simulation scenarios by assuming both direct and mutational additive genetic sources of variation

\begin{tabular}{|c|c|c|c|c|}
\hline \multirow[b]{2}{*}{ Item $^{1}$} & \multicolumn{2}{|c|}{ Standard heritability $^{2}$} & \multicolumn{2}{|c|}{ Mutational heritability $^{3}(\times 100)$} \\
\hline & Mean & Range & Mean & Range \\
\hline \multicolumn{5}{|l|}{ Scenario G3 } \\
\hline $\mathrm{h}^{2}=0.10$ & 0.12 & $0.04-0.21$ & 0.04 & $0.01-1.57$ \\
\hline $\mathrm{h}^{2}=0.25$ & 0.24 & $0.13-0.35$ & 0.09 & $0.02-2.30$ \\
\hline $\mathrm{h}^{2}=0.50$ & 0.48 & $0.37-0.66$ & 0.14 & $0.04-2.75$ \\
\hline \multicolumn{5}{|l|}{ Scenario G5 } \\
\hline $\mathrm{h}^{2}=0.10$ & 0.12 & $0.05-0.16$ & 0.04 & $0.02-1.50$ \\
\hline $\mathrm{h}^{2}=0.25$ & 0.23 & $0.17-0.31$ & 0.11 & $0.04-1.99$ \\
\hline $\mathrm{h}^{2}=0.50$ & 0.52 & $0.43-0.66$ & 0.19 & $0.07-2.32$ \\
\hline \multicolumn{5}{|l|}{ Scenario G10 } \\
\hline $\mathrm{h}^{2}=0.10$ & 0.11 & $0.06-0.15$ & 0.06 & $0.02-0.91$ \\
\hline $\mathrm{h}^{2}=0.25$ & 0.25 & $0.19-0.29$ & 0.14 & $0.07-1.09$ \\
\hline $\mathrm{h}^{2}=0.50$ & 0.51 & $0.42-0.58$ & 0.25 & $0.13-0.89$ \\
\hline
\end{tabular}

${ }^{1} \mathrm{G} 3$, G5, and $\mathrm{G} 10=3,5$, and 10 generations, respectively.

${ }^{2} \mathrm{~h}_{a}^{2}=\sigma_{a}^{2} / \sigma_{T}^{2}$, where $\sigma_{a}^{2}$ is the additive variance and $\sigma_{T}^{2}$ is the total phenotypic variance.

${ }^{3} \mathrm{~h}_{m}^{2}=\sigma_{m}^{2} / \sigma_{T}^{2}$, where $\sigma_{m}^{2}$ is the mutational variance and $\sigma_{T}^{2}$ is the total phenotypic variance.

moderate-to-high heritabilities or deeper pedigrees took benefit of the most complex structure of model AM accounting for new mutational variability. Similar evidences were revealed by $\mathrm{r}_{u, \hat{u}}$, where higher accuracies $(P$ $<0.05)$ were reported for model AM under simulation scenarios with moderate-to-high heritabilities or deeper pedigrees (Tables 3 and 4), albeit the gains were marginal. These results suggested that model AM could be ineffective to properly capture mutational variability when lowly heritable traits were analyzed on shallow pedigrees, whereas heritability and pedigree depth showed synergistic behavior in less extreme scenarios [i.e., traits with moderate-to-high heritabilities (even under shallow pedigrees) or moderate-to-deeper pedigrees (even for lowly heritable traits) would marginally benefit from the inclusion of new mutational effects in GBLUP models].

Regardless of the GBLUP parameterization, $r_{u, \hat{u}}$ showed very similar trends when comparing different heritabilities, pedigree depths, or individuals with and without phenotypic data. The accuracy of the genomic predictions significantly increased with heritability as anticipated by Calus et al. (2008), and demonstrated by previous studies without the inclusion of mutational effects (Meuwissen, 2009; Zhong et al., 2009). Accuracy was higher for phenotyped individuals than for those without contributing phenotypic data to the analysis. Indeed, this decline in precision for the unphenotyped individuals from recent generations has been widely demonstrated in the scientific literature (Meuwissen

Table 2. Average deviance information criterion (DIC) estimates (means $\pm \mathrm{SE}$ )

\begin{tabular}{lccc}
\hline Item $^{1}$ & Model $\mathrm{A}^{2}$ & Model AM & Difference $^{4}$ \\
\hline Scenario G3 & & & \\
$\mathrm{h}^{2}=0.10$ & $4,807 \pm 4$ & $4,815 \pm 4$ & $-8 \pm 2^{* *}$ \\
$\mathrm{~h}^{2}=0.25$ & $4,829 \pm 4$ & $4,832 \pm 4$ & $-3 \pm 3^{\text {NS }}$ \\
$\mathrm{h}^{2}=0.50$ & $4,877 \pm 5$ & $4,867 \pm 4$ & $10 \pm 4^{*}$ \\
Scenario G5 & & & \\
$\mathrm{h}^{2}=0.10$ & $7,431 \pm 5$ & $7,429 \pm 5$ & $2 \pm 3^{\mathrm{NS}}$ \\
$\mathrm{h}^{2}=0.25$ & $7,472 \pm 5$ & $7,462 \pm 5$ & $22 \pm 3^{*}$ \\
$\mathrm{~h}^{2}=0.50$ & $7,501 \pm 6$ & & \\
Scenario G10 & & $13,183 \pm 8$ & $9 \pm 4^{*}$ \\
$\mathrm{~h}^{2}=0.10$ & $13,192 \pm 8$ & $13,216 \pm 8$ & $20 \pm 4^{* * *}$ \\
$\mathrm{~h}^{2}=0.25$ & $13,236 \pm 9$ & $13,225 \pm 9$ & $35 \pm 5^{* * *}$ \\
$\mathrm{~h}^{2}=0.50$ & $13,260 \pm 9$ & & \\
\hline
\end{tabular}

${ }^{1} \mathrm{G} 3$, G5, and $\mathrm{G} 10=3,5$, and 10 generations, respectively.

${ }^{2}$ Genomic BLUP (GBLUP) model accounting for standard additive genetic effects.

${ }^{3}$ Genomic BLUP model accounting for both standard additive and new mutational genetic effects.

${ }^{4}$ Differences between model A and model AM were tested by a paired-samples $t$-test.

${ }^{*} P<0.05 ;{ }^{* *} P<0.01 ;{ }^{* * *} P<0.001$. 
Table 3. Correlation coefficients $(\times 100$; mean \pm SE) between simulated and predicted breeding values for individuals with phenotypic data

\begin{tabular}{llll}
\hline Item $^{1}$ & Model $\mathrm{A}^{2}$ & Model AM $^{3}$ & Difference $^{4}$ \\
\hline Scenario G3 & & & \\
$\mathrm{h}^{2}=0.10$ & $52.49 \pm 0.23$ & $52.51 \pm 0.22$ & $0.02 \pm 0.05^{\mathrm{NS}}$ \\
$\mathrm{h}^{2}=0.25$ & $64.25 \pm 0.24$ & $64.29 \pm 0.25$ & $0.04 \pm 0.04^{\mathrm{NS}}$ \\
$\mathrm{h}^{2}=0.50$ & $82.19 \pm 0.24$ & $82.27 \pm 0.26$ & $0.08 \pm 0.05^{\mathrm{NS}}$ \\
Scenario G5 & & & \\
$\mathrm{h}^{2}=0.10$ & $57.66 \pm 0.24$ & $57.69 \pm 0.25$ & $0.03 \pm 0.04^{\mathrm{NS}}$ \\
$\mathrm{h}^{2}=0.25$ & $69.21 \pm 0.25$ & $85.38 \pm 0.23$ & $0.10 \pm 0.04^{*}$ \\
$\mathrm{~h}^{2}=0.50$ & $85.12 \pm 0.23$ & $63.10 \pm 0.23$ & $0.26 \pm 0.03^{* * *}$ \\
Scenario G10 & & $74.03 \pm 0.25$ & $0.07 \pm 0.05^{\mathrm{NS}}$ \\
$\mathrm{h}^{2}=0.10$ & $63.03 \pm 0.22$ & $89.17 \pm 0.18$ & $0.24 \pm 0.04^{* * *}$ \\
$\mathrm{~h}^{2}=0.25$ & $73.81 \pm 0.21$ & & $0.57 \pm 0.03^{* * *}$ \\
$\mathrm{~h}^{2}=0.50$ & $88.60 \pm 0.19$ &
\end{tabular}

${ }^{1} \mathrm{G} 3$, G5, and $\mathrm{G} 10=3,5$, and 10 generations, respectively.

${ }^{2}$ Genomic BLUB (GBLUP) model accounting for standard additive genetic effects.

${ }^{3}$ Genomic BLUP model accounting for both standard additive and new mutational genetic effects.

${ }^{4}$ Differences between model A and model AM were tested by a paired-samples $t$-test.

${ }^{*} P<0.05 ;{ }^{* * *} P<0.001$.

et al., 2001; Legarra et al., 2008). It is important to note that $\mathrm{r}_{u, \hat{u}}$ increased with the number of generations involved in $\mathrm{G}$, this being probably due to the growth of the genomic/phenotypic data set (Meuwissen et al., 2001; Goddard, 2009) and the rise of the degree of relationship among individuals from late generations in small populations (Habier et al., 2007).

In summary, maximum DIC and $\mathrm{r}_{u, \hat{u}}$ differences were reported in scenario G10 and $\mathrm{h}^{2}=0.5$. The accuracy of genomic breeding values increased $0.57 \%$ (phenotyped individuals) or $1.07 \%$ (unphenotyped individuals) under model AM. This suggests that model AM could be of interest for genomic selection in livestock populations, although each case must be evaluated on its merits, depending on population structure and the genetic model underlying the trait. Moreover, other genetics-related phenomena such as selection, population stratification, or even genetic drift must be carefully considered because they could vary the real contribution of mutational effects.

A delicate point is the consideration of generations when they overlap. Wray (1990) pointed out that the decisive notion in her model was time span, not generations; the model applies equally well to overlapping generations pedigrees. In this case, generations would be cohorts born in the same time span (e.g., year-byyear groups). The only requirement is that no individual within one cohort can be descendant from another from the same cohort. Even in our model using genomic relationships, a notion of pedigree or time flow is absolutely

Table 4. Correlation coefficients $(\times 100$; mean $\pm \mathrm{SE})$ between simulated and predicted breeding values for individuals without phenotypic data

\begin{tabular}{|c|c|c|c|}
\hline Item $^{1}$ & Model A ${ }^{2}$ & Model $\mathrm{AM}^{3}$ & Difference $^{4}$ \\
\hline \multicolumn{4}{|l|}{ Scenario G3 } \\
\hline $\mathrm{h}^{2}=0.10$ & $37.40 \pm 0.51$ & $37.39 \pm 0.50$ & $-0.01 \pm 0.06^{\mathrm{NS}}$ \\
\hline $\mathrm{h}^{2}=0.25$ & $50.40 \pm 0.51$ & $50.44 \pm 0.52$ & $0.04 \pm 0.05^{\mathrm{NS}}$ \\
\hline $\mathrm{h}^{2}=0.50$ & $66.70 \pm 0.52$ & $66.80 \pm 0.53$ & $0.10 \pm 0.05^{*}$ \\
\hline \multicolumn{4}{|l|}{ Scenario G5 } \\
\hline $\mathrm{h}^{2}=0.10$ & $40.92 \pm 0.49$ & $40.97 \pm 0.48$ & $0.05 \pm 0.5^{\mathrm{NS}}$ \\
\hline $\mathrm{h}^{2}=0.25$ & $55.76 \pm 0.50$ & $55.89 \pm 0.50$ & $0.13 \pm 0.06^{*}$ \\
\hline $\mathrm{h}^{2}=0.50$ & $73.03 \pm 0.49$ & $73.44 \pm 0.50$ & $0.43 \pm 0.06^{* * *}$ \\
\hline \multicolumn{4}{|l|}{ Scenario G10 } \\
\hline $\mathrm{h}^{2}=0.10$ & $59.52 \pm 0.50$ & $59.68 \pm 0.50$ & $0.16 \pm 0.07^{*}$ \\
\hline $\mathrm{h}^{2}=0.25$ & $66.99 \pm 0.49$ & $67.61 \pm 0.49$ & $0.62 \pm 0.06^{* * *}$ \\
\hline $\mathrm{h}^{2}=0.50$ & $79.96 \pm 0.49$ & $81.03 \pm 0.50$ & $1.07 \pm 0.05^{* * *}$ \\
\hline
\end{tabular}

${ }^{1} \mathrm{G} 3$, G5, and $\mathrm{G} 10=3,5$, and 10 generations, respectively.

${ }^{2}$ Genomic BLUP (GBLUP) model accounting for standard additive genetic effects.

${ }^{3}$ Genomic BLUP model accounting for both standard additive and new mutational genetic effects.

${ }^{4}$ Differences between model A and model AM were tested by a paired-samples $t$-test.

${ }^{*} P<0.05 ;{ }^{* * *} P<0.001$. 
necessary, because mutations are transmitted forward but not backward. Consider thus cohorts $i=1,2$, . . . , $q$ as the starting point. According to properties of G (Gianola et al., 2009) and under Hardy-Weinberg equilibrium, $\mathbf{G}_{i}$ would contain an average of 1 in the diagonal and 0 off-diagonal for individuals from cohort $i$. Within this context, we could reduce the notion of cohort to the extreme where each individual defines a new cohort and, therefore, $\mathbf{G}_{m}=\mathbf{G}_{1}+\mathbf{G}_{2}+\ldots+$ $\mathbf{G}_{t}$, where $t$ is the total number of individuals in the pedigree file. Only descendants of the $i$ th individual would have values different from 0 in $\mathbf{G}_{i}$, as previously suggested by García-Cortés et al. (2010) for matrix A. For this partitioning of $\mathbf{G}_{m}$, the diagonal element of individual $i$ in $\mathbf{G}_{i}$ is fixed to 0 , and this is so because the covariance matrix $\mathbf{G}_{i}$ must be centered around this element. The $\mathbf{G}_{-}$-specific mutational genetic value of $i$ is exactly defined as 0, has no uncertainty, and this individual becomes the genetic basis of the genetic covariance matrix $\mathbf{G}_{i}$. However, the $i$ th element in matrix $\mathbf{G}_{m}$ will not be 0 because of the $i$ th diagonal contributions of remaining cohorts.

To the best of our knowledge, this is the first approach contributing a reliable way to account for new additive mutational variability in genomic prediction models, with this GBLUP parameterization being able to provide small advantages in terms of accuracy of the genomic predictions, a way of handling mutational variance in selection schemes, and possibly more accurate estimates of mutational variance than those based on pedigrees. Although the relevance of new mutations could be small when individuals from few generations are involved (i.e., first steps in genomic selection programs), this model could be of special relevance as times goes on and genotyped individuals accumulate generation by generation. Also, it allows better handling of new genetic variability, such as the selection of "original" animals or loci, which are penalized by standard animal breeding techniques (Goddard, 2009).

\section{ACKNOWLEDGMENTS}

The research contract of J. Casellas was partially financed by Spain's Ministerio de Ciencia e Innovación (program Ramón y Cajal, reference RYC-2009-04049). This research was funded by grant AGL2010-21176/ GAN (Ministerio de Ciencia e Innovación, Madrid, Spain).

\section{REFERENCES}

Calus, M. P., T. H. E. Meuwissen, A. P. de Roos, and R. F. Veerkamp. 2008. Accuracy of genomic selection using different methods to define haplotypes. Genetics 178:553-561.
Casellas, J. 2011. Accounting for new mutations in the genomic relationship matrix. J. Anim. Sci. 89(E-Suppl. 1):535.

Casellas, J., and J. F. Medrano. 2008. Within-generation mutation variance for litter size in inbred mice. Genetics 179:2147-2155.

Casellas, J., and L. Varona. 2011. Effect of mutation age on genomic predictions. J. Dairy Sci. 94:4224-4229.

García-Cortés, L. A., J. C. Martínez-Ávila, and M. A. Toro. 2010. Fine decomposition of the inbreeding and the coancestry coefficients by using the tabular method. Conserv. Genet. 11:1945-1952.

Gianola, D., G. de los Campos, W. G. Hill, E. Manfredi, and R. Fernando. 2009. Additive genetic variability and the Bayesian alphabet. Genetics 183:347-363.

Goddard, M. 2009. Genomic selection: Prediction of accuracy and maximisation of long term response. Genetica 136:245-257.

Habier, D., R. L. Fernando, and J. C. M. Dekkers. 2007. The impact of genetic relationship information on genome-assisted breeding values. Genetics 177:2389-2397.

Henderson, C. R. 1984. Applications of Linear Models in Animal Breeding. University of Guelph, Guelph, ON, Canada.

Hickey, J. M., and G. Gorjanc. 2012. Simulated data for genomic selection and genome-wide association studies using a combination of coalescent gene drop methods. G3 (Bethesda) 2:425-427.

Houle, D., B. Morikawa, and M. Lynch. 1996. Comparing mutational variabilities. Genetics 143:1467-1483.

Legarra, A., C. Robert-Granié, E. Manfredi, and J.-M. Elsen. 2008. Performance of genomic selection in mice. Genetics 180:611-618.

Lynch, M. 1988. The rate of polygenic mutation. Genet. Res. 51:137148 .

Maher, B. 2008. Personal genomes: The case of the missing heritability. Nature 456:18-21.

Meuwissen, T. H. E. 2009. Accuracy of breeding values of 'unrelated' individuals predicted by dense SNP genotyping. Genet. Sel. Evol. $41: 35$.

Meuwissen, T. H. E., B. J. Hayes, and M. E. Goddard. 2001. Prediction of total genetic value using genome-wide dense marker maps. Genetics 157:1819-1829.

Nejati-Javaremi, A., C. Smith, and J. P. Gibson. 1997. Effect of total allelic relationship on accuracy of evaluation and response to selection. J. Anim. Sci. 75:1738-1745.

Raftery, A. E., and S. M. Lewis. 1992 How many iterations in the Gibbs sampler? Pages 763-773 in Bayesian Statistics IV. J. M. Bernardo, J. O. Berger, A. P. Dawid, and A. F. M. Smith, ed. Oxford University Press, Oxford, UK.

Spiegelhalter, D. J., N. G. Best, B. P. Carlin, and A. van der Linder. 2002. Bayesian measures of model complexity and fit. J. R. Stat. Soc., B 64:583-639.

Toro, M. Á., L. A. García-Cortés, and A. Legarra. 2011. A note on the rationale for estimating genealogical coancestry from molecular markers. Genet. Sel. Evol. 43:27.

VanRaden, P. M. 2008. Efficient methods to compute genomic predictions. J. Dairy Sci. 91:4414-4423.

VanRaden, P. M., C. P. Van Tassell, G. R. Wiggans, T. S. Sonstegard, R. D. Schnabel, J. F. Taylor, and F. S. Schenkel. 2009. Reliability of genomic predictions for North American Holstein bulls. J. Dairy Sci. 92:16-24.

Wray, N. R. 1990. Accounting for mutation effects in the additive genetic variance-covariance matrix and its inverse. Biometrics 46:177-186.

Zhong, S., J. C. M. Dekkers, R. L. Fernando, and J.-L. Jannink. 2009. Factors affecting accuracy from genomic selection in populations derived from multiple inbred lines: A barley case study. Genetics 182:355-364. 